\title{
The Significance of Motor Evoked Potential Changes and Utility of Multimodality Intraoperative Monitoring in Spinal Surgery: A Retrospective Analysis of Consecutive Cases at a Single Institution
}

\author{
Joseph N. Frazzetta $^{1}$, Ryan C. Hofler ${ }^{2}$, William Adams ${ }^{1}$, Michael J. Schneck ${ }^{2}$, G. Alexander Jones ${ }^{2}$ \\ 1. Neurosurgery, Loyola University Stritch School of Medicine, Maywood, USA 2. Neurosurgery, Loyola University \\ Medical Center, Maywood, USA
}

Corresponding author: G. Alexander Jones, joneshvna@gmail.com

\section{Abstract \\ Objective}

The objective of the study is to identify specific population groups that may benefit from intraoperative motor evoked potentials (MEP) and to assess positive predictive value (PPV) and negative predictive value (NPV) changes during operation by correlating these with postoperative motor outcomes.

\section{Methods}

We retrospectively reviewed 1,043 consecutive patient cases undergoing spine surgery with and without intraoperative monitoring (IOM) at a single institution from January 1, 2016 to December 31, 2017. Demographic and clinical outcome data were collected at multiple time points. An MEP amplitude decrease of $50 \%$ or greater was correlated with a motor deficit for this study.

\section{Results}

On multivariate analysis, patients with coronary artery disease and who received IOM were more likely to experience no new deficit $(\mathrm{p}=0.047)$ than those who did not receive IOM. Additionally, patients with hyperlipidemia and coronary artery disease (CAD) were less likely than those without to experience no new deficit ( $\mathrm{p}=0.001$ and $\mathrm{p}=0.02$, respectively). MEP accounted for 244 cases, of which 15 had alert MEP criteria but no deficit for a PPV of $21.05 \%$ at day 1 post-operation. Day 7-30 PPV declined to $14.29 \%$, and by day 90 , there was no association.

\section{Conclusion}

Review began 10/09/2020 Review ended 12/08/2020 Published 12/13/2020

\section{(c) Copyright 2020}

Frazzetta et al. This is an open access article distributed under the terms of the Creative Commons Attribution License CC-BY 4.0., which permits unrestricted use, distribution, and reproduction in any medium, provided the original author and source are credited.
Among patients in our study with CAD, IOM use was associated with significantly better outcomes. Patients with higher intraoperative blood loss, hyperlipidemia, and those with CAD were at increased risk of new neurological deficit. The use of motor evoked potentials was associated with low sensitivity and low PPV.

Categories: Neurology, Neurosurgery

Keywords: intraoperative neuromonitoring, motor evoked potential, positive predictive value, spine surgery

\section{Introduction}

Neurological complications during spine surgery are rare and include direct spinal cord trauma and cord ischemia. Such events are life-changing and can result in muscle weakness, pain, and even paralysis. The rate of intraoperative complications ranges from 0-3\%. However, in spine surgeries of increased risk, such as intradural spinal cord tumors or spine deformity cases, intraoperative complications can be seen at much higher rates [1-4]. Historically, the method to detect intraoperative spinal cord injury was the Stagnara wake-up test, which required anesthetic reversal to observe gross motor function [5]. The subsequent introduction of intraoperative monitoring (IOM) has allowed for earlier detection of irritation and damage of neural elements.

IOM comprises three main categories: motor-evoked potentials (MEP), somatosensory-evoked potentials (SSEP), and electromyography (EMG) [6, 7]. Recent literature has not clearly affirmed the value of IOM during spine surgery, nor the degree to which neurological outcomes are improved as a result of its use [811]. Most research on IOM focuses on the sensitivity and specificity of these modalities; however, the positive predictive value (PPV) and negative predictive value (NPV) may better characterize the information provided by IOM. The purpose of this study is to present a single-center, retrospective review of consecutive spine operations, with the goals of identifying specific patient groups that may benefit from multimodality 
IOM; and specifically assessing the utility of MEP changes during operation as predictors of postoperative motor outcomes via PPV and NPV.

\section{Materials And Methods \\ Data collection}

This study was approved by the Institutional Review Board. Patient consent was not required for this retrospective chart review. All patients undergoing spine surgery at a single institution between January 1 , 2016 and December 31, 2017 were included. Patients were identified via the operative case log by one author who did not participate in the care of these individuals. We identified 968 patients undergoing 1,043 procedures and separated them into two groups based on whether or not IOM was used. Demographic data were collected, along with the following variables: site of operation; the number of spine levels operated on; anterior vs. posterior approach; indications for operation; comorbidities; IOM use and which modalities used (SSEP, MEP, and/or EMG); and preoperative and postoperative neurologic outcome data at several time points. Patients with incomplete preoperative data, infant spine cases (under two years of age at time of operation), and patients without data from at least one postoperative visit were excluded from analysis (Figure 1).

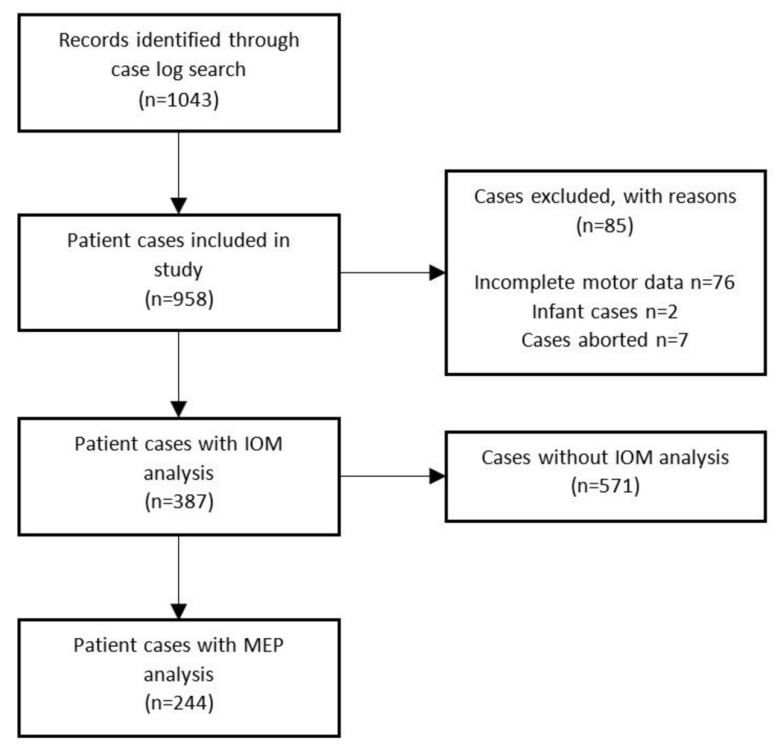

FIGURE 1: Flowsheet with excluded patients

\section{Monitoring}

A motor evoked potential (MEP) amplitude decrease of $50 \%$ or greater is the alert threshold at our institution. By protocol, the operating surgeon is notified of the change, and this is documented in the IOM report. We identified all alerts and correlated these with new onset motor deficit at multiple points postoperatively. We separately studied patients with a sustained MEP change, i.e., one which did not return to at least $50 \%$ of the baseline amplitude before the end of the operation. Somatosensory evoked potentials (SSEP) and electromyography (EMG) data were included in our analysis as well.

\section{Statistical analysis}

Summary frequencies were displayed as valid counts and proportions for all baseline demographics by intraoperative status. Univariable generalized linear mixed effects models were used to determine the odds of having no new postoperative neurological deficit (defined here as a 'good neurological outcome'), as a function of treatment characteristics and risk factors. For these models, a binomial distribution with logit link was specified for the motor score, and each model allowed an interaction term to estimate stratified effect sizes at 1, 7-30, and 90 days post-surgery.

A follow-up multivariable generalized linear mixed effects models was used to estimate the adjusted odds of a good neurological outcome as a function of covariates selected because of their significance on univariable analysis or improvement in model fit statistics. As before, a binomial distribution with logit link was specified for motor score, and this model included an interaction term to allow the association between intraoperative monitoring and the odds of good neurological outcome to depend on patients' coronary 


\section{Cureus}

artery disease status. Because patients could contribute multiple procedures to the analysis, random intercepts were allowed for each patient to account for this dependency; a Kenward-Roger correction was used to adjust the denominator degrees of freedom [12].

Sensitivity, specificity, PPV and NPV were estimated with exact confidence limits based on the relationship between an alert threshold change in MEP during operation and the presence of a new post-operative motor deficit. A true positive was defined as a patient with both an MEP alert and new post-operative deficit. A true negative was defined as a patient with no MEP alert and no new post-operative deficit. A false positive was a patient with an MEP alert but no new post-operative deficit, and a false negative was a patient with no MEP alert who experienced a new post-operative deficit. All analyses were completed using SAS version 9.4 (SAS Institute, Cary, USA).

\section{Results}

\section{Patient characteristics, univariable analysis}

A total of 968 patients were identified, undergoing 1,043 operations, and attending 3,129 postoperative visits. Among these, 894 patients undergoing 958 procedures had recorded motor examinations prior to their operation, and for at least one day postoperatively. Of these procedures, 387 (40\%) underwent some form of IOM (including 244 with MEP), while 571 (60\%) underwent an operation without IOM (Table 1). IOM was more likely to be utilized in cases with existing preoperative neurological deficit, cervical or thoracic location, myelopathy, scoliosis or deformity correction, trauma, tumor, and hypertension. IOM was less likely to be used in patients with lumbar location and radiculopathy. 


\begin{tabular}{|c|c|c|c|c|c|c|c|}
\hline & \multicolumn{6}{|c|}{ Intraoperative monitoring } & \multirow{3}{*}{$\mathbf{p}$} \\
\hline & \multicolumn{2}{|c|}{ No $(n=571)$} & \multicolumn{2}{|c|}{ Yes $(n=387)$} & \multicolumn{2}{|c|}{ Total $(\mathrm{N}=958)$} & \\
\hline & Count & $\%$ & Count & $\%$ & Count & $\%$ & \\
\hline Male & 305 & $53.40 \%$ & 212 & $54.80 \%$ & 517 & $54.00 \%$ & \\
\hline Female & 266 & $46.60 \%$ & 175 & $45.20 \%$ & 441 & $46.00 \%$ & \\
\hline Pre-operative motor deficit & 214 & $37.50 \%$ & 191 & $49.40 \%$ & 405 & $42.30 \%$ & $<0.001$ \\
\hline New post-operative motor deficit & 38 & $6.70 \%$ & 39 & $10.10 \%$ & 77 & $8.00 \%$ & 0.06 \\
\hline Cervical location & 126 & $22.10 \%$ & 202 & $52.30 \%$ & 328 & $34.30 \%$ & $<0.001$ \\
\hline Thoracic location & 77 & $13.50 \%$ & 148 & $38.30 \%$ & 225 & $23.50 \%$ & $<0.001$ \\
\hline Lumbar location & 401 & $70.20 \%$ & 154 & $39.90 \%$ & 555 & $58.00 \%$ & $<0.001$ \\
\hline Anterior approach & 110 & $19.30 \%$ & 82 & $21.20 \%$ & 192 & $20.10 \%$ & 0.45 \\
\hline Posterior approach & 478 & $83.70 \%$ & 314 & $81.30 \%$ & 792 & $82.80 \%$ & 0.56 \\
\hline Myelopathy & 124 & $21.70 \%$ & 192 & $49.60 \%$ & 316 & $33.00 \%$ & $<0.001$ \\
\hline Radiculopathy & 300 & $52.50 \%$ & 144 & $37.20 \%$ & 444 & $46.30 \%$ & $<0.001$ \\
\hline Deformity / scoliosis & 26 & $4.60 \%$ & 42 & $10.90 \%$ & 68 & $7.10 \%$ & $<0.001$ \\
\hline Neurogenic Claudication & 93 & $16.30 \%$ & 69 & $17.80 \%$ & 162 & $16.90 \%$ & 0.53 \\
\hline Trauma & 51 & $8.90 \%$ & 58 & $15.00 \%$ & 109 & $11.40 \%$ & 0.005 \\
\hline Tumor & 8 & $1.40 \%$ & 17 & $4.40 \%$ & 25 & $2.60 \%$ & 0.01 \\
\hline Diabetes & 131 & $22.90 \%$ & 73 & $18.90 \%$ & 204 & $21.30 \%$ & 0.19 \\
\hline Hypertension & 275 & $48.20 \%$ & 214 & $55.30 \%$ & 489 & $51.00 \%$ & 0.048 \\
\hline Coronary artery disease & 62 & $10.90 \%$ & 55 & $14.20 \%$ & 117 & $12.20 \%$ & 0.18 \\
\hline Hyperlipidemia & 169 & $29.60 \%$ & 133 & $34.40 \%$ & 302 & $31.50 \%$ & 0.16 \\
\hline
\end{tabular}

\section{TABLE 1: Baseline demographics by intraoperative monitoring status}

The average age for the non-intraoperative monitoring group $(M=56.22, S D=15.98)$ was comparable to the average age for the intraoperative monitoring group $(M=58.58, S D=17.38 ; p=0.12)$. For the whole sample, the average baseline age was $57.18(S D=16.59)$. The distribution of blood loss (cc) for the non-intraoperative monitoring group ( $M d n=50.00$, IQR: 20.00-250.00) was lower than the distribution of blood loss (cc) for the intraoperative monitoring group (Mdn=200.00, IQR: 75.00-500.00; $p<0.001$ ). For the whole sample, the median blood loss (cc) was 100.00 (IQR: 25.00-300.00).

$\mathrm{N}$ - number of procedures; IQR - interquartile range

On univariable analysis (Table 2 and Figure 2), patients undergoing IOM were less likely to experience a good neurological outcome, though this conclusion was not statistically significant at day $1(\mathrm{p}=0.07)$, day 7$30(\mathrm{p}=0.28)$, or day $90(\mathrm{p}=0.07)$. Conversely, on the first postoperative day, patients with a lumbar location were 1.64 times more likely to have a good neurological outcome than those without a lumbar location (95\% CI: 1.01 - 2.66, p=0.047), and patients with neurogenic claudication were 2.53 (95\% CI: 1.06 - 6.04) times more likely to have a good outcome than those without neurogenic claudication $(\mathrm{p}=0.04)$. Patients with a tumor were less likely than those without a tumor to have a good neurological outcome on the first postoperative day ( $\mathrm{OR}=0.33$; $95 \% \mathrm{CI}: 0.11$ - 0.99; $\mathrm{p}=0.048$ ).

\begin{tabular}{|c|c|c|c|c|}
\hline \multirow{2}{*}{ Days } & \multirow{2}{*}{ Odds ratio } & \multicolumn{2}{|c|}{$95 \%$ confidence interval } & \multirow{2}{*}{$\mathbf{p}$} \\
\hline & & Lower & Upper & \\
\hline \multicolumn{5}{|c|}{ Intraoperative monitoring: Yes vs. No } \\
\hline Day 1 & 0.637 & 0.393 & 1.034 & 0.068 \\
\hline
\end{tabular}




\section{Cureus}

Day $7-30$

Day 90

Pre-operative motor deficit: Yes vs. No

Day 1

Day $7-30$

Day 90

Age (per 1-year increase)

Day 1

Day $7-30$

Day 90

Sex: male vs. female

Day 1

Day $7-30$

Day 90

Cervical location: Yes vs. No

Day 1

Day $7-30$

Day 90

Thoracic location: Yes vs. No

Day 1

Day $7-30$

Day 90

Lumbar location: Yes vs. No

Day 1

Day $7-30$

Day 90

Anterior approach: Yes vs. No

Day 1

Day $7-30$

Day 90

Posterior approach: Yes vs. No

Day 1

Day $7-30$

Day 90

Myelopathy: Yes vs. No

Day 1

Day $7-30$

Day 90

Radiculopathy: Yes vs. No

Day 1
0.769

0.544

0.872

0.834

0.656

1.005

0.986

0.981

0.974

1.003

2.003

0.633

0.715

0.741

0.813

0.775

1.083

1.636

1.28

1.478

1.133

1.545

1.202

0.979

0.591

0.982

0.636

0.567

0.816

1.096
0.477

0.284

0.537

0.518

0.343

0.991

0.972

0.96

0.6

0.624

1.024

0.388

0.439

0.383

0.47

0.461

0.493

1.007

0.796

0.773

0.608

0.777

0.531

0.515

0.28

0.432

0.389

0.349

0.419

0.674

0.067

0.176

0.374

1.435

1.406

0.459

0.336

0.843

2.38

2.656

0.047

0.308

0.238

2.826

2.111

0.694

3.073

0.215

2.718

0.659

0.949

0.167

0.966

2.233

1.041

0.072

0.022

0.549

1.59 


\section{Cureus}

Day $7-30$

0.981

Day 90

Deformity / scoliosis: Yes vs No

Day 1

Day $7-30$

Day 90

Neurogenic claudication: Yes vs. No

Day 1

Day $7-30$

Day 90

Trauma: Yes vs. No

Day 1

Day $7-30$

Day 90

Tumor: Yes vs. No

Day 1

Day $7-30$

Day 90

Diabetes: Yes vs. No

Day 1

Day $7-30$

Day 90

Hypertension: Yes vs. No

Day 1

Day $7-30$

Day 90

Coronary artery disease: Yes vs. No

Day 1

Day $7-30$

Day 90

Hyperlipidemia: Yes vs No

Day 1

Day $7-30$

Day 90

Monitorable: Yes vs No

Day 1

Day $7-30$

Day 90

Estimated blood loss (per 100cc increase)

1.18

1.361

0.25

0.87

0.42

0.631
0.611

1.577

1.059

0.938

0.074

1.998

0.822

2.534

1.614

0.818

0.945

0.998

4.897

0.333

0.806

0.669

1.204

1.076

0.717

1.112

0.657

0.714

0.767

0.332

0.802

0.539

6.696

0.262

1.87

0.639

4.095

0.794

0.34

1.063

6.037

0.036

0.813

3.204

0.172

0.371

1.804

0.618

0.446

2.003

0.882

2.073

0.996

36.826

0.123

0.651

0.112

0.989

0.048

0.294

6.302

0.693

0.046

1.369

0.11

0.458

1.418

0.453

1.145

0.142

2.729

0.657

0.664

1.744

0.767

1.157

0.173

2.122

0.746

0.583

0.34

1.272

0.213

0.448

1.692

0.682

0.185

0.957

0.039

0.433

1.177

0.186

0.47

1.251

0.288

0.172

0.641

0.001

0.389

1.024

0.062

0.497

1.292

0.364

0.282

1.033

0.062 


\section{Cureus}

\begin{tabular}{|c|c|c|c|c|}
\hline Day 1 & 1.043 & 0.97 & 1.122 & 0.255 \\
\hline Day $7-30$ & 0.944 & 0.901 & 0.99 & 0.017 \\
\hline Day 90 & 0.987 & 0.917 & 1.063 & 0.735 \\
\hline
\end{tabular}

TABLE 2: Odds of no new deficit

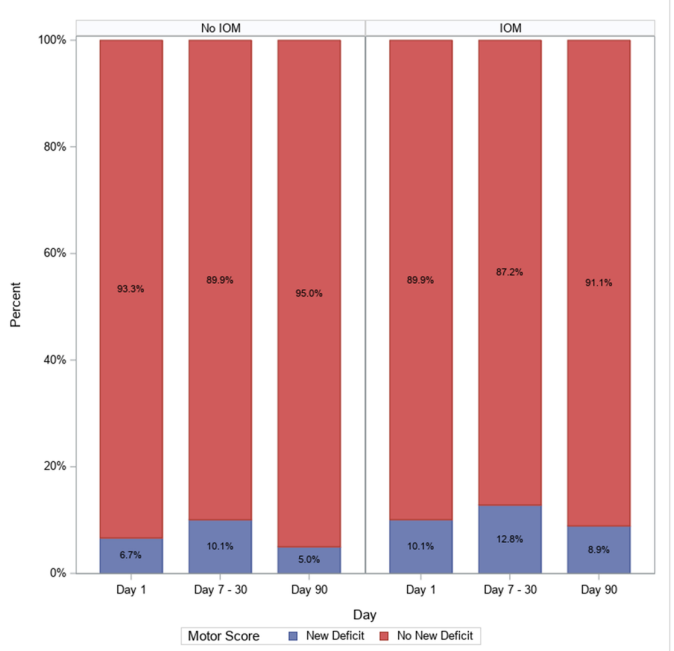

FIGURE 2: Outcomes breakdown at multiple post-operative points, with and without intraoperative monitoring

On postoperative days 7-30, each additional 100cc intraoperative blood loss correlated with a $5.6 \%$ decrease in the odds of a good neurological outcome ( $\mathrm{OR}=0.94,95 \% \mathrm{CI}$ : 0.90 - 0.99; $\mathrm{p}=0.02$ ), and patients with myelopathy were only 0.57 ( $95 \%$ CI: 0.35 - 0.92) times as likely to experience a good outcome as those without myelopathy $(\mathrm{p}=0.02)$.

On postoperative day 90 , males were more likely than females to experience a good outcome ( $O R=2.00,95 \%$ CI: $1.02-3.91 ; \mathrm{p}=0.04)$. Patients with coronary artery disease were less likely to have a good outcome $(\mathrm{OR}=0.42$, 95\% CI: 0.19 - 0.96; $\mathrm{p}=0.04$ ), as were patients with hyperlipidemia (OR=0.33, 95\% CI: 0.17 - 0.64; $\mathrm{p}=0.001$ ).

\section{Patient characteristics, multivariable analysis}

On multivariable analysis, patients with coronary artery disease who received IOM were 2.59 (95\% CI: 1.01 6.65) times more likely than those who did not receive IOM to experience a good neurological outcome $(\mathrm{p}=0.047$, Table 3). Patients with hyperlipidemia were significantly less likely than those without hyperlipidemia to experience a good outcome ( $\mathrm{OR}=0.65,95 \%$ CI: $0.45-0.93$; $\mathrm{p}=0.02)$. 


\section{Cureus}

\begin{tabular}{|c|c|c|c|c|}
\hline & \multirow{2}{*}{ Adjusted odds ratio } & \multicolumn{2}{|c|}{$95 \%$ confidence interval } & \multirow{2}{*}{$\mathbf{p}$} \\
\hline & & Lower & Upper & \\
\hline \multicolumn{2}{|l|}{$I^{\prime O M}{ }^{*} \mathrm{CAD}$ interaction } & & & 0.02 \\
\hline IOM: Yes versus No | CAD = Yes & 2.594 & 1.012 & 6.645 & 0.047 \\
\hline IOM: Yes versus No $\mid \mathrm{CAD}=$ No & 0.883 & 0.529 & 1.475 & 0.63 \\
\hline CAD: Yes versus No| IOM = Yes & 1.450 & 0.724 & 2.904 & 0.29 \\
\hline CAD: Yes versus No| IOM = No & 0.494 & 0.269 & 0.906 & 0.02 \\
\hline \multicolumn{2}{|l|}{ Day } & & & 0.02 \\
\hline Day 7-30 versus Day 1 & 0.721 & 0.517 & 1.006 & 0.054 \\
\hline Day 90 versus Day 1 & 1.218 & 0.817 & 1.817 & 0.33 \\
\hline Day 90 versus Day 7-30 & 1.689 & 1.134 & 2.516 & 0.01 \\
\hline Claudication: Yes versus No & 2.242 & 1.379 & 3.644 & 0.001 \\
\hline Hyperlipidemia: Yes versus No & 0.646 & 0.451 & 0.926 & 0.02 \\
\hline
\end{tabular}

\section{TABLE 3: Adjusted odds of no new deficit}

Valid $\mathrm{N}=966$. The multivariable estimates are also adjusted for physician type (ortho vs. neuro).

IOM - intraoperative monitoring (IOM); CAD - coronary artery disease

The odds of a good outcome were significantly higher by postoperative day 90 than postoperative day 7-30 $(\mathrm{OR}=1.69,95 \% \mathrm{CI}: 1.13-2.52 ; \mathrm{p}=0.01)$; that is, patients with a deficit at postoperative day $7-30$ saw this deficit resolve by postoperative day 90 . Those with claudication were significantly more likely than those without claudication to experience a good outcome (OR=2.24, 95\% CI: $1.38-3.64 ; \mathrm{p}=0.001)$.

\section{Intraoperative MEP changes}

A total of 244 cases with at least one day of follow-up were available to assess the sensitivity, specificity, negative predictive value, and positive predictive value of an MEP alert change to detect a new deficit (Table 4). Among these patients, the prevalence of a new deficit on the first post-operative day was $12.7 \%(n=31)$, and only four (1.6\%) were true positive cases leading to imprecise estimates of sensitivity (12.90\%; $95 \%$ CI: 3.63 - 29.83) and PPV (21.05\%; 95\% CI: 6.05\% - 45.57\%). Conversely, 198 (81\%) patients were true negative cases leading to estimates of specificity $(92.96 \%$; $95 \%$ CI: 88.65\% - 96.01\%) and NPV (88.00\%; 95\% CI: $83.02 \%-91.94 \%)$. 


\section{Cureus}

\begin{tabular}{|c|c|c|c|c|}
\hline & \multirow{2}{*}{ Valid $\mathbf{N}$} & \multirow{2}{*}{ Estimate } & \multicolumn{2}{|c|}{ Exact $95 \%$ confidence interval } \\
\hline & & & Lower & Upper \\
\hline Day 1 & 244 & & & \\
\hline Sensitivity & & 12.90 & 3.63 & 29.83 \\
\hline Specificity & & 92.96 & 88.65 & 96.01 \\
\hline Positive predictive value & & 21.05 & 6.05 & 45.57 \\
\hline Negative predictive value & & 88.00 & 83.02 & 91.94 \\
\hline False positive probability & & 78.95 & 54.43 & 93.95 \\
\hline False negative probability & & 12.00 & 8.06 & 16.98 \\
\hline Day 7-30 & 203 & & & \\
\hline Sensitivity & & 7.14 & 0.88 & 23.50 \\
\hline Specificity & & 93.14 & 88.33 & 96.41 \\
\hline Positive predictive value & & 14.29 & 1.78 & 42.81 \\
\hline Negative predictive value & & 86.24 & 80.50 & 90.81 \\
\hline False positive probability & & 85.71 & 57.19 & 98.22 \\
\hline False negative probability & & 13.76 & 9.19 & 19.50 \\
\hline Day 90 & 162 & & & \\
\hline Sensitivity & & 0.00 & -- & -- \\
\hline Specificity & & 93.10 & 87.68 & 96.64 \\
\hline Positive predictive value & & 0.00 & -- & -- \\
\hline Negative predictive value & & 88.82 & 82.70 & 93.35 \\
\hline False positive probability & & 100.00 & 69.15 & 100.00 \\
\hline False negative probability & & 11.18 & 6.65 & 17.30 \\
\hline
\end{tabular}

\section{TABLE 4: Epidemiology scores for motor evoked potential change and post-operative motor}

scores

Valid $\mathrm{N}$ - the number of patients used to calculate the estimates. These epidemiology scores are restricted to patients' first surgery.

By day 7-30, 203 patients had follow-up data. The prevalence of a new deficit was $13.8 \%(\mathrm{n}=28)$, and only two $(1.0 \%)$ were identified as true positive cases yielding imprecise estimates of sensitivity $(7.14 \%$; $95 \% \mathrm{CI}$ : $0.88 \%$ - 23.50\%) and PPV (14.29\%; 95\% CI: $1.78 \%$ - 42.81\%). As before, a large number of patients ( $\mathrm{n}=163$ or $80 \%$ ) were true negative cases, yielding estimates of specificity (93.14\%; $95 \%$ CI: $88.33 \%-96.41 \%$ ) and NPV (86.24\%; 95\% CI: 80.50\% - 90.81\%).

Finally, 162 patients had follow-up data available by day 90 . For these patients, the prevalence of a new deficit was $10 \%(n=17)$, and there were no true positive cases. However, $135(83 \%)$ patients were true negative cases leading to estimates of specificity (93.10; 95\% CI: 87.68 - 96.64) and NPV (88.82\%; 95\% CI: 82.70 - 93.35).

Of all patients with MEP change during operation, only three had an amplitude drop that persisted until the end of the operation. Among these three patients, none had a postoperative motor deficit, rendering the sensitivity and PPV zero for a sustained MEP dropout during the operation (Table 5). 


\section{Cureus}

\begin{tabular}{|c|c|c|c|c|}
\hline & \multirow{2}{*}{ Valid $\mathbf{N}$} & \multirow{2}{*}{ Estimate } & \multicolumn{2}{|c|}{ Exact $95 \%$ confidence interval } \\
\hline & & & Lower & Upper \\
\hline Day 1 & 228 & & & \\
\hline Sensitivity & & 0.00 & -- & -- \\
\hline Specificity & & 98.51 & 95.70 & 99.69 \\
\hline Positive predictive value & & 0.00 & -- & -- \\
\hline Negative predictive value & & 88.00 & 83.02 & 91.94 \\
\hline False positive probability & & 100.00 & 29.24 & 100.00 \\
\hline False negative probability & & 12.00 & 8.06 & 16.98 \\
\hline Day 7-30 & 191 & & & \\
\hline Sensitivity & & 0.00 & -- & -- \\
\hline Specificity & & 98.79 & 95.69 & 99.85 \\
\hline Positive predictive value & & 0.00 & -- & -- \\
\hline Negative predictive value & & 86.24 & 80.50 & 90.81 \\
\hline False positive probability & & 100.00 & 15.81 & 100.00 \\
\hline False negative probability & & 13.76 & 9.19 & 19.50 \\
\hline Day 90 & 153 & & & \\
\hline Sensitivity & & 0.00 & -- & -- \\
\hline Specificity & & 99.26 & 95.97 & 99.98 \\
\hline Positive predictive value & & 0.00 & -- & -- \\
\hline Negative predictive value & & 88.82 & 82.70 & 93.35 \\
\hline False positive probability & & 100.00 & 2.50 & 100.00 \\
\hline False negative probability & & 11.18 & 6.65 & 17.30 \\
\hline
\end{tabular}

TABLE 5: Epidemiology scores for persistent motor evoked potential change and post-operative motor scores

Valid $\mathrm{N}$ - the number of patients used to calculate the estimates. These epidemiology scores are restricted to patients' first surgery.

\section{Discussion}

\section{Patient characteristics and use of IOM}

The use of monitoring at our institution is at the discretion of the operating surgeon. In general, it is more likely to be used in cases with a higher risk of neurological injury, including myelopathy, tumor, and deformity, and less likely in cases of isolated lumbar stenosis. Thus, our finding that patients undergoing IOM were more likely to have a new neurological deficit than those without IOM, while not statistically significant, must be interpreted considering this selection bias: surgeons preferentially monitor the cases at higher risk of deficit. This also accounts for better outcomes in patients with lumbar stenosis and neurogenic claudication.

Our results suggest that coronary artery disease is not only associated with increased risk of postoperative deficit but that patients in this group with IOM fared better than those without. Additionally, hyperlipidemia and increasing blood loss were risk factors for the new neurological deficit. Given the methodology of our study and the lack of physiologic rationale to support them, we think it is best to assume these findings are spurious.

It is no surprise that neurologic deficits were more common at day 1 and days 7-30 following operation than at day 90. A number of neurologic deficits, including C5 palsy, will improve with time. 


\section{Intraoperative MEP changes}

Ideally, IOM will allow a surgeon to perform a safer operation by alerting the surgeon of impending or early neurological compromise before it leads to a permanent deficit. The best hope for this, in our opinion, is the use of motor evoked potentials (MEP), which provide real-time feedback on the function of the corticospinal tracts at the level of the spinal cord.

For this modality, our data were very disappointing. On postoperative day 1, we saw new deficits in 31 out of 244 patients. However, only four of these had MEP changes during the operation, yielding a sensitivity of $12.9 \%$. For all 19 patients with an MEP change during the operation, the PPV was just $21.05 \%$. The PPV dropped to $14.29 \%$ at day 7-30, and zero by day 90 . While we expected the PPV to be low and the NPV high (indicating a high number of false positive tests), we were surprised that the sensitivity was so low, especially considering that our alert threshold is an amplitude drop of just $50 \%$, while many others use $80 \%$, $90 \%$, or even $100 \%[13,14]$.

The statistics we choose to analyze are really at the core of this analysis. Many other series report on the sensitivity and specificity of MEP changes during operation. However, for two reasons, these numbers are less relevant to the operating surgeon than PPV and NPV.

First, sensitivity and specificity are easier concepts to grasp but generally less useful than PPV and NPV. Still, sensitivity and specificity have some role in helping a clinician to decide what diagnostic test to order before ordering the test when testing for a disease with high prevalence. The ideal screening test for a given disease, for example, will have high sensitivity and low cost. It can be widely deployed, will accurately identify everyone who has (or might have) a disease, and do so at a low cost. For such a screening test, specificity is much less important. However, a confirmatory test with high specificity is necessary to exclude the false positives generated by the screening test.

A change in MEP is a different matter than a screening test. In this case, the surgeon is presented with the result of a test and needs to know how to interpret this; PPV and NPV are better suited to help the surgeon understand the given result than sensitivity and specificity. The ideal test would have 100\% PPV, meaning all positive results are true positives. Applied to IOM, this means any MEP drop during the case would indicate a true neurological insult and would not be caused by anesthetic factors, room temperature, positional ischemia or neuropraxia, or any other spurious input. It would also have $100 \% \mathrm{NPV}$, with all negative results being true negatives. This means that any patient without MEP change during the operation would emerge from the operation with no new deficit. Unfortunately, a low PPV, as we found in this series, means that most MEP alerts are false alarms. Stating the initial PPV of $21.05 \%$ another way, nearly four out of five patients with an intraoperative drop in MEP awoke with no new deficit whatsoever. In each case, a great deal of time (and no small amount of stress for the surgeon and OR team) is given over to treat a 'problem' that really doesn't exist.

Second, PPV and NPV are dependent on the prevalence of a condition, whereas sensitivity and specificity are not. The lower the prevalence of the condition (in this case, a new neurological deficit), the lower the PPV. In this series, the risk of new neurologic deficit drops to $10.5 \%$ by 90 days (including a number of new C5 palsies); with such a low prevalence, the PPV will be low as well. Understanding the interdependence of PPV and prevalence is very important when interpreting results (such as an MEP drop) in a test designed to screen for something of very low prevalence (new neurologic deficit after the operation).

This might argue for using MEPs only during the cases with the highest risk (i.e., the highest prevalence) of postoperative neurologic deficit, which should lead to a higher PPV and thus greater utility for the surgeon. However, the existing literature has not shown a clear benefit to monitoring in cases such as spinal cord tumor resection [15-17].

Our study, and the larger body of literature on this topic, clearly highlight the need for a prospective trial to determine the utility of IOM. However, several challenges must be overcome.

For any topic to warrant the effort and expense of a prospective trial, whether randomized or single-arm, there must exist clinical equipoise regarding the treatment. IOM carries additional burdens in terms of cost, and duration of the operation, and of anesthesia. The payoff for this, at least in theory, is to allow the surgeon a chance to prevent an iatrogenic neurological injury or correct a temporary insult before it becomes a permanent injury. This benefit remains elusive, at least in the literature published to date. We think equipoise really does exist in the field of IOM.

Further challenges for a prospective trial exist. The blinding of the surgeon to the results of data being collected in real time raises serious ethical questions. Additionally, logistical issues involving the monitoring technician and interpreting physician being sent away on the morning of an operation when the patient is randomized to the control arm could be problematic.

The best solution might be a single-arm trial, looking only at patients undergoing IOM. The focal point of 
this would be identifying cases in which a loss of MEP signals can be reversed by the surgeon and/or the anesthesiologist, ideally following a standardized, and predetermined, protocol. This data could be used to calculate the cost of IOM on the basis of each injury avoided and the number needed to treat (NNT) to prevent one injury. However, the validity of such a study depends on at least the high sensitivity of IOM, which remains an open question.

\section{Limitations}

The primary limitation of this study is its retrospective design. IOM use was based on the attending surgeon's discretion, leading to potential selection bias and heterogeneity in terms of which cases they chose to monitor or not. Also, we do not have a mechanism to identify which cases had an intraoperative MEP drop that was successfully addressed and rectified by the surgeon. The sensitivity was low enough that this was not a critical matter in data analysis. However, distinguishing between MEP alerts due to neural element compromise and those due to anesthetic or other factors would be important in any prospective trial.

\section{Conclusions}

Our study demonstrates the significance of an intraoperative MEP change when considered in the context of the low prevalence of true neural element injury. The use of motor evoked potentials (MEP) to predict or prevent neurological injury was associated with low sensitivity and low PPV on postoperative day 1 ; by postoperative day 90, there was no correlation between MEP change and motor outcome. Additionally, we have identified specific patient groups who may benefit from IOM. Patients with coronary artery disease had a higher risk of a new postoperative neurological deficit than those without. Among patients with CAD, the use of IOM was associated with significantly better outcomes. Patients with higher intraoperative blood loss and those with hyperlipidemia were at increased risk of new neurological deficit. This paper adds to our understanding of the strengths and limitations of IOM and further illustrates the need for a prospective trial to clarify the value of IOM in spine surgery.

\section{Additional Information \\ Disclosures}

Human subjects: Consent was obtained by all participants in this study. Kenneth Craig Micetich issued approval 210574. This study was approved by the Institutional Review Board. Patient consent was not required for this retrospective chart review. Animal subjects: All authors have confirmed that this study did not involve animal subjects or tissue. Conflicts of interest: In compliance with the ICMJE uniform disclosure form, all authors declare the following: Payment/services info: All authors have declared that no financial support was received from any organization for the submitted work. Financial relationships: All authors have declared that they have no financial relationships at present or within the previous three years with any organizations that might have an interest in the submitted work. Other relationships: This article was previously accepted for publication by another journal, and subsequently withdrawn at our request.

\section{References}

1. Ahn H, Fehlings MG: Prevention, identification, and treatment of perioperative spinal cord injury . Neurosurg Focus. 2008, 25:e15. 10.3171/foc.2008.25.11.e15

2. Bible JE, Rihn JA, Lim MR, Brodke DS, Lee JY: Avoiding and managing intraoperative complications during cervical spine surgery. J Am Acad Orthop Surg. 2015, 23:81-90. 10.5435/jaaos-d-14-00446

3. Matsuyama Y, Sakai Y, Katayama Y, et al.: Surgical results of intramedullary spinal cord tumor with spinal cord monitoring to guide extent of resection. J Neurosurg Spine. 2009, 10:404-413. 10.3171/2009.2.spine08698

4. Yoshida G, Hasegawa T, Yamato Y, et al.: Predicting perioperative complications in adult spinal deformity surgery using a simple sliding scale. Spine. 2018, 43:562-570. 10.1097/brs.0000000000002411

5. Vauzelle C, Stagnara P, Jouvinroux P: Functional monitoring of spinal cord activity during spinal surgery . Clin Orthop Relat Res. 1973, 93:173-178. 10.1097/00003086-197306000-00017

6. Fehlings MG, Brodke DS, Norvell DC, Dettori JR: The evidence for intraoperative neurophysiological monitoring in spine surgery: does it make a difference?. Spine. 2010, 35:37-46. 10.1097/BRS.0b013e3181d8338e

7. Lall RR, Hauptman JS, Munoz C, et al.: Intraoperative neurophysiological monitoring in spine surgery: indications, efficacy, and role of the preoperative checklist. Neurosurg Focus. 2012, 33:e10. 10.3171/2012.9.focus12235

8. Thirumala PD, Muralidharan A, Loke YK, Habeych M, Crammond D, Balzer J: Value of intraoperative neurophysiological monitoring to reduce neurological complications in patients undergoing anterior cervical spine procedures for cervical spondylotic myelopathy. J Clin Neurosci. 2016, 25:27-35. 10.1016/j.jocn.2015.06.027

9. Traynelis VC, Abode-Iyamah KO, Leick KM, Bender SM, Greenlee JD: Cervical decompression and reconstruction without intraoperative neurophysiological monitoring. J Neurosurg Spine. 2012, 16:107-113. 10.3171/2011.10.spine11199

10. Raynor BL, Padberg AM, Lenke LG, et al.: Failure of intraoperative monitoring to detect postoperative neurologic deficits: a 25-year experience in 12,375 spinal surgeries. Spine. 2016, 41:1387-1393. 10.1097/brs.0000000000001531 


\section{Cureus}

11. Resnick DK, Anderson PA, Kaiser MG, et al.: Electrophysiological monitoring during surgery for cervical degenerative myelopathy and radiculopathy. J Neurosurg Spine. 2009, 11:245-252.

10.3171/2009.2.spine08730

12. Kenward M, Roger J: Improved approximation to the precision of fixed effects from restricted maximum likelihood. Comput Stat Data Anal. 2009, 53:2583-2595. 10.1016/j.csda.2008.12.013

13. Koht A, Sloan TB: Intraoperative monitoring: recent advances in motor evoked potentials. Anesthesiol Clin. 2016, 34:525-535. 10.1016/j.anclin.2016.04.006

14. Scibilia A, Terranova C, Rizzo V, et al.: Intraoperative neurophysiological mapping and monitoring in spinal tumor surgery: sirens or indispensable tools?. Neurosurg Focus. 2016, 41:e18. 10.3171/2016.5.focus16141

15. Harel R, Schleifer D, Appel S, Attia M, Cohen ZR, Knoller N: Spinal intradural extramedullary tumors: the value of intraoperative neurophysiologic monitoring on surgical outcome. Neurosurg Rev. 2017, 40:613-619. 10.1007/s10143-017-0815-2

16. Kahraman S, Gocmen S, Gokmen MHA, Acka G, Pusat S: Intraoperative neurophysiologic monitoring for lumbar intradural schwannomas: does it affect clinical outcome?. World Neurosurg. 2019, 124:e789-e792. 10.1016/j.wneu.2019.01.054

17. Lakomkin N, Mistry AM, Zuckerman SL, et al.: Utility of Intraoperative Monitoring in the Resection of Spinal Cord Tumors: An Analysis by Tumor Location and Anatomical Region. Spine. 2018, 53:287-294. 10.1097/brs.0000000000002300 